\title{
Need to talk to someone about acerediftation?
}

ACRL determined several months ago to prepare a directory of individuals who enjoy expertise in accreditation, both institutional and specialized. Accordingly, an Accreditation Advisors Board was formed.

\section{Criteria}

This Board solicited applications from individu als who had accreditation experience and met the following criteria:

1. An AlA-accredited degree in library/information science.

2. Knowledge of academic librarianship, its role in higher education, and the accreditation process.

3. Interpersonal, communication, and organizational skills.

4. Served as a member on three or more "accreditation teams" for a regional accrediting association (e.g., Middle States, Southern, Western, etc.) or professional association (e.g., National Council for Accreditation of Teacher Edu- cation [NCATE], Accreditation Board of Engineering and Technology, etc.).

\section{How to become an advisor}

There were several responses and those who have met the criteria, as of this writing, are listed below. Should you have any questions related to the list please contact any member of the Advisory Board including Mignon Adams (Philadelphia College of Pharmacy \& Science), Keith Cottam (University of Wyoming), Barbara Williams-Jenkins (South Carolina State College), or David Walch (California Polytechnic University). If you meet the criteria and are willing to be listed as an accreditation consultant you should request an application from: David B. Walch, Dean of Library Services, Kennedy Library, California Polytechnic State University, San Luis Obispo, CA 93407; (805) 756-2344.

Ed. note: For more information on accreditation be sure to see the articles on pages $442-47$.

\section{- ACRL Approval Plans ... S Save Time and Money!
ACRL Publicatis ns Provide Practical Ideas, Tools and Methods for:
- Management - Bibliographic Instruction - Special Collections \\ - Collection Development - Statistics \& Research

$\frac{\frac{\text { Association OF }}{\text { COLLEGE }}}{\frac{\text { \& RESEARCH }}{\text { LIBRARIES }}}$ \\ The convenient and cost-cutting ACRL approval order plan provides automatic priority shipping of ACRL's new books at a $20 \%$ discount to ACRL members ( $10 \%$ to non-members). \\ There are two approval plan categories from which to chooset \\ Plan P guarantees that you will automatically be sent all new ACRL publications* including CLIP Notes. ACRL publishes approximately 5 to 6 new titles each year. \\ -Periodicals and subscription items are not included \\ You may change or cancel your approval order at any time. Retum privíeges guaranteed. \\ Enrollment Form \\ a Plan P guatantees shipment of all new non-periodical ACRL. publications including CLIP Noles. \\ Please give your ALA membership number if you are eligible for the 20\%, ACRL membership discount. Note: Personal memberships may not be used to qualify institutions for the ACRL member discount. \\ ALA membership number \\ P.O. $=$ \\ Ship to: \\ Bill no: \\ Plan PC is exclusively for titles in the DLIP Notes (College Library Information Packets) series. CLIP Notes collect data and sample documents from academic libraries to assist librarians in establishing or refining services and opc rations.}

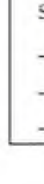

$$
440
$$




\section{COA}

Joanne R. Euster

University Librarian

University of California, Irvine

P.O. Box 19557

Irvine, CA 92713

Phone: (714) 856-8926

Fax: (714) $725-2472$

E-mail: Euster@uci.edu

\section{MIDDLE STATES}

Caroline M. Coughlin

Director, Univ. Lib.

Drew University

36 Madison Avenue

Madison, N.J. 07940

Phone: (201) 408-3322

Fax: (201) 408-3770

E-mail: CCOUGHLI@DREW.DREW.EDU

\section{Antoinette M. Kania}

Dean of Libraries

Suffolk Community College

533 College Road

Selden, NY 11784

Phone: (516) 451-4174

Fax: (516) 451-4697

\section{MIDDLE STATES/NEW ENGLAND}

Jay K. Lucker

53 Buckman Drive

Lexington, MA 02173-6019

Phone: (617) 253-5651

Fax: (617) 253-8894

E-mail: LUCKER@MITUMA.MIT.Edu

\section{NORTH CENTRAL}

Ronald G. Leach

4815 Wolftree Drive

Terre Haute, IN 47805

Phone: (812) 237-3700

Fax: (812) 237-3376

E-mail: LIBLECH@INDST.INDSTATE.EDU

\section{NORTH CENTRAL/SOUTHERN}

Edward D. Garten

Director of Univ, Lib.

The University of Dayton

300 College Park

Dayton, $\mathrm{OH} 45469-1360$

Phone: (513) 229-4265

Fax: (513) $229-4590$

E-mail: Bitnet.Garten@dayton

\section{SOUTHERN}

Joseph F. Lindenfeld

1850 Peabody Avenue

Memphis, TN 38104-4025

Phone: (901) 725-8824

\author{
Marcia J. Myers \\ Director of Libraries \\ Eastern Kentucky University \\ 135 Lakeshore Drive \\ Richmond, KY 40475 \\ Phone: (615) 974-4465 \\ Fax: (615) 622-1174
}

James F. Parks Jr.

(SACS) MS. Comm. on College

Coliege Librarian

Millsaps College

P.O. Box 150148

Jackson, MS 39210-0001

Phone: (601) 974-1070

Fax: (601) 974-1082

E-mail: PARKS@OKRAO1.MILLSAPS.EDU

Tom G. Watson

ALA Comm. on Accreditation

Vice-President for Univ. Rel.

The University of the South

University Avenue

Sewanee, TN 37375

Phone: (615) 598-1213

Fax: (615) 598-1199

Delmus E. Williams

Dean of Univ. Libraries

University of Akron

Bierce Library

Akron, OH 44325-1706

Phone: (216) 972-7495

Fax: (216) 972-6383

E-mail: WILLIAMS@UAKRON.EDU

Joan $H$. Worley

Director, Lamar Memorial Library

Maryville College

Maryville, TN 37801

Phone: (615) 981-8257

Fax: (615) 981-8170

\section{WESTERN}

David B. Walch

Dean of Library Services

California Polytechnic Univ.

San Luis Obispo, CA 93407

Phone: (805) 756-2344

Fax: (805) 756-1415

E-mail: DU525@OASIS.CALPOLY.EDU

David C. Weber

Director Emeritus

863 Lathrop Drive

Stanford, CA 94305

Phone: (415) 857-9219

E-mail: CM.DCW@STANFORD.BITNET 